ARTICLE

https://doi.org/10.1038/s41467-020-14546-z

\title{
DNA repair by Rad52 liquid droplets
}

\author{
Roxanne Oshidari 10 1, Richard Huang ${ }^{1}$, Maryam Medghalchi ${ }^{1,2}$, Elizabeth Y.W. Tse ${ }^{3}$, Nasser Ashgriz ${ }^{2}$, \\ Hyun O. Lee ${ }^{3,4}$, Haley Wyatt ${ }^{3,4}$ \& Karim Mekhail (1) 1,4*
}

Cellular processes are influenced by liquid phase separation, but its role in DNA repair is unclear. Here, we show that in Saccharomyces cerevisiae, liquid droplets made up of DNA repair proteins cooperate with different types of DNA damage-inducible intranuclear microtubule filaments (DIMs) to promote the clustering of DNA damage sites and maintain genome stability. Rad52 DNA repair proteins at different DNA damage sites assemble in liquid droplets that fuse into a repair centre droplet via the action of petite DIMs (pti-DIMs). This larger droplet concentrates tubulin and projects short aster-like DIMs (aster-DIMs), which tether the repair centre to longer DIMs mediating the mobilization of damaged DNA to the nuclear periphery for repair. Our findings indicate that cooperation between Rad52 liquid droplets and various types of nuclear filaments promotes the assembly and function of the DNA repair centre.

\footnotetext{
${ }^{1}$ Department of Laboratory Medicine and Pathobiology, Faculty of Medicine, University of Toronto, MaRS Centre, 661 University Ave., Toronto, ON M5G 1M1, Canada. ${ }^{2}$ Multiphase Flow and Phase Systems Laboratory, Department of Mechanical and Industrial Engineering, Faculty of Applied Science and Engineering, University of Toronto, 5 King's College Circle, M5S 3 G8 Toronto, ON, Canada. ${ }^{3}$ Department of Biochemistry, Faculty of Medicine, University of Toronto, MaRS Centre, 661 University Ave., Toronto, ON M5G 1M1, Canada. ${ }^{4}$ Canada Research Chairs Program, Faculty of Medicine, University of Toronto, 1 King's College Circle, M5S 1A8 Toronto, ON, Canada. *email: karim.mekhail@utoronto.ca
} 
E ukaryotic genomes are dynamic structures and are nonrandomly arranged within the cell nucleus, which is defined by an envelope perforated with nuclear pore complexes (NPCs) ${ }^{1}$. Genome dynamics allow cells to repair DNA doublestrand breaks (DSBs), which are highly toxic DNA lesions that trigger the DNA damage checkpoint ${ }^{2}$. Specifically, the movement of DSBs allows them to escape repair-repressive heterochromatin domains, search for homologous sequences or localise to repairconducive $\mathrm{NPCs}^{3-15}$.

The de novo assembly of intranuclear filaments, onto which DSBs are transported by motor proteins, promotes DSB escape from heterochromatin or movement to NPCs ${ }^{6,16-18}$. In S. cerevisiae cells with a single DSB, the Kinesin-14 motor proteins Kar3 and Cik1 associate with the break site and are required for its capture by long DNA damage-inducible intranuclear microtubule filaments (DIMs), which emanate from the microtubuleorganizing centre (MTOC) 6,16 . The break is then directionally mobilised by Kinesin-14 along a DIM and moved away from the MTOC to NPCs for repair ${ }^{16}$. Similarly, in cells treated with carcinogens such as methyl methanesulfonate (MMS), damaged DNA, identified by the presence of the Rad52 DNA repair protein, moves along DIMs to NPCs, where the focus later dissolves, marking repair completion ${ }^{16}$. In flies, a similar actin/myosinbased mechanism moves DSBs for repair ${ }^{8,18}$. Importantly, in a given cell, carcinogens can trigger several DSBs that co-localise and create a DNA repair centre, which is enriched in Rad52 in yeast but remains poorly understood across eukaryotes ${ }^{17,19}$. The forces driving DSB clustering, whether such forces crosstalk with nuclear filaments, and how clustering promotes genome stability remain unclear.

Here, by using a combination of live-cell imaging, in vitro assays and in silico simulations, we demonstrate that the DNA repair centre is created through the fusion of liquid-like bodies of Rad52 protein marking different DSB sites within the nucleus. This fusion is driven by cooperation between distinct types of intranuclear microtubule filaments. Disrupting the filaments limits fusion and increases genome instability. Rad52 mutants that fail to form liquid-like bodies in vitro, fail to efficiently form repair centres or maintain genome stability in vivo. Taken together, our results decipher DNA repair centre assembly and function.

\section{Results}

Cells with Rad52 foci exhibit different types of DIMs. We first aimed to closely examine the relationship between DIMs and DSBs within the nuclei of live cells. Therefore, we used a yeast system for the fluorescence-based visualisation of DSB-indicating Rad52, a-tubulin Tub1 and NPC-indicating Nup49 protein ${ }^{16}$ (Supplementary Fig. 1a, b). Cells treated with MMS exhibited Rad52/RPA-positive DSBs (Supplementary Fig. 1c). MMS induced one DIM in cells containing a single large and bright Rad52 focus (Fig. 1a, b). DIMs emanated from the MTOC and efficiently captured the large Rad52 focus, as expected (Fig. 1c) ${ }^{16}$. In contrast, the MTOC of cells containing more than one Rad52 focus tended to exhibit several shorter microtubule filaments (denoted petite DIMs or pti-DIMs) that failed to capture damaged DNA (Fig. 1a, c). Thus, cells with several DSB-indicating Rad52 foci exhibit several pti-DIMs, which, in contrast to the DIM in cells with one large Rad52 focus, fail to capture the Rad52 foci.

Rad52 foci exhibit liquid-like properties. Strikingly, in cells harbouring one or more Rad52 foci, the foci exhibited liquid-like properties $^{20-22}$. First, in pti-DIM-positive cells, Rad52 foci that were induced using the genotoxic agents MMS or zeocin engaged in dripping, fusion, or bumping encounters with each other (Fig. 1d; Supplementary Fig. 1d; Supplementary Movies 1-3).
Second, in DIM-positive cells, the Rad52 focus exhibited wetting behaviour consisting of focus flattening against the nuclear envelope or the DIM (Fig. 1e). Third, Rad52 foci were abrogated by the liquid droplet disruptor 1,6-Hexanediol, which did not disrupt the overall nuclear localisation of Rad52 (Supplementary Fig. 1e $)^{2}$. Lastly, Rad52 foci quickly lost signal during fluorescence loss in photobleaching (FLIP), confirming that the foci constituents are liquid-liquid phase-separated but undergo exchange with the surrounding nucleoplasm (Fig. 1f; Supplementary Fig. 1f; Supplementary Movie 4). The data indicate that Rad52 foci exhibit liquid-like properties in vivo.

Rad52 disorder and phase separation in genome stability. $S$. cerevisiae Rad52 purified from Escherichia coli phase separated from buffer and formed liquid droplets at low salt concentrations $(\text { Fig. } 2 \mathrm{a}-\mathrm{c})^{23,24}$. These droplets were spherical, often fused with each other, and were disrupted by 1,6-Hexanediol (Fig. 2d; Supplementary Movie 5; Supplementary Fig. 2a). Consistent with its liquid droplet-forming capacity, Rad52 is predicted to exhibit a high level of intrinsic disorder (Supplementary Fig. 2b). In fact, a Rad52 mutant $(\Delta 307)$ lacking a portion of the disordered domain failed to phase separate in vitro (Fig. 2e, f). Importantly, upon DNA damage induction in cells expressing $\Delta 307$, the percentage of cells with repair foci decreases from $\sim 55 \%$ to $\sim 5 \%$, the remaining foci-positive cells also exhibit a higher number of smaller foci, and the whole-cell population is hypersensitive to DNA damage ${ }^{25}$. In addition, in strains expressing $\Delta 307$ but not wild-type Rad52, sensitivity to MMS can be partly rescued following the overexpression of $\operatorname{Rad} 51^{25}$. This indicates that $\Delta 307$ is defective in focus formation in vivo and fails to phase separate in vitro, but has the ability to promote Rad51 loading and strand exchange in vivo. Furthermore, within our experimental conditions, addition of the heterotrimeric repair factor RPA in complex with ssDNA did not alter Rad52 phase separation (Supplementary Fig. 2c, d) ${ }^{26}$. While these data show that Rad52 has an intrinsic ability to assemble liquid droplets, in vitro conditions likely do not fully recapitulate the in vivo environment in which Rad52 phase separates.

We then asked whether 1,6-Hexanediol, which represses Rad52 droplets in vivo (Supplementary Fig. 1e), hyper-induces the DNA damage checkpoint, which is indicated by the phosphorylation of Rad53 (CHK2 tumour suppressor in mammals) ${ }^{2}$. Only in MMStreated cells, 1,6-Hexanediol hyper-induced Rad53 phosphorylation, and this without altering cellular Rad52 levels (Supplementary Fig. 3a, b). Thus, 1,6-Hexanediol hinders genome stability only upon DNA damage induction by disrupting Rad52 phase separation or potentially other factors in the cell.

pti-DIMs promote Rad52 droplet fusion and genome stability. In vivo, a single engineered DSB induces one Rad52 focus and one long DIM but no pti-DIMs, suggesting their potential role in DSB clustering ${ }^{16}$. Indeed, in MMS-treated cells, pti-DIMs engaged in extension-shortening cycles with velocities correlating with Rad52 droplet velocities (Fig. 3a, b; Supplementary Movies 6 and 7). Computational fluid dynamics (CFD) simulations can reveal whether and how a velocity-induced flow drives the fusion of two liquid droplets ${ }^{20}$. Indeed, CFD simulations incorporating parameters observed in vivo during Rad52 droplet fusion events revealed that pti-DIM dynamics may be able to generate flows that lower the pressure between Rad52 droplets, driving their fusion (Fig. 3c, f; Supplementary Fig. 4a, b; Supplementary Movies 8 and 9). Fusion occurred only at droplet viscosities $\leq 0.005 \mathrm{~Pa}$ s and was very efficient when the pti-DIM velocity was applied at a $90^{\circ}$ angle to the axis connecting two droplets. Therefore, the in silico simulations, which are predictive 
a

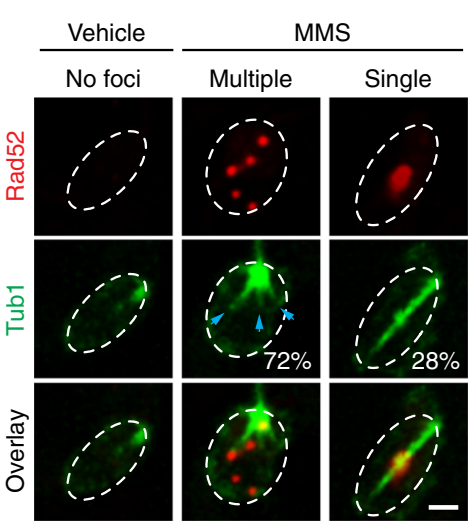

b

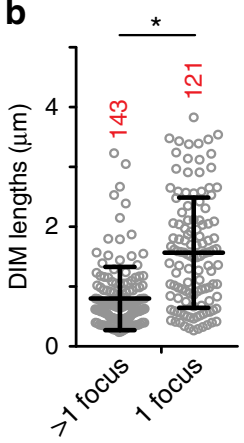

C

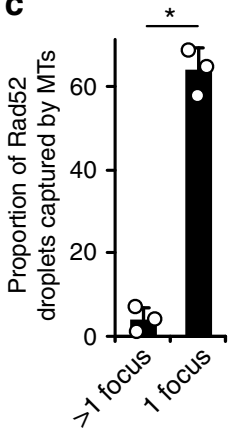

d

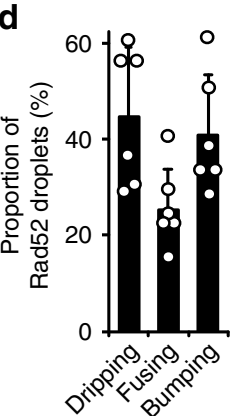

$0 \mathrm{~s} \rightarrow 59 \mathrm{~s} \rightarrow 118 \mathrm{~s}$

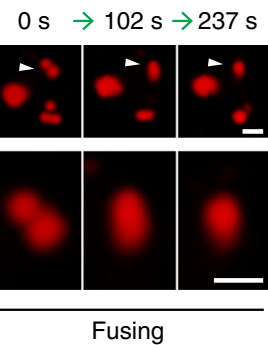

$0 s \rightarrow 30 s \rightarrow 60 s$
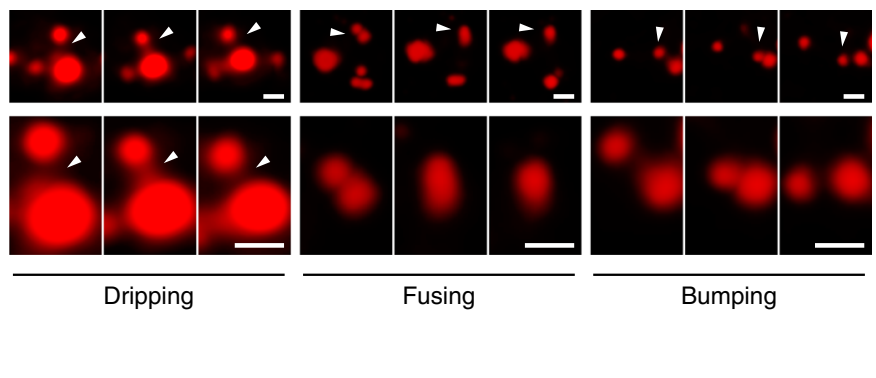

e
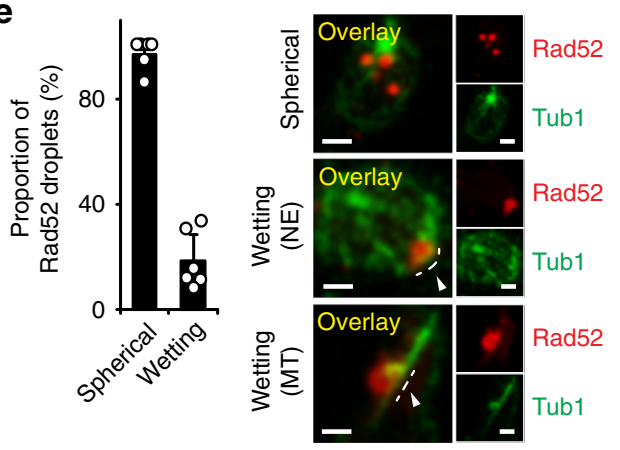

f

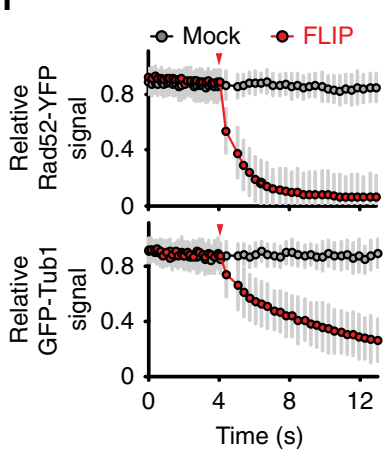

Fig. 1 DSB-indicating Rad52 forms liquid droplets in vivo. a-c Live-cell microscopy shows that nuclei with $>1$ Rad52 foci (a) exhibit shorter microtubule filaments $(\mathbf{b})$ that cannot capture the foci $(\mathbf{c})(n=3)$. In $\mathbf{a}$, shown are percentage of cells with given DIM species and pti-DIM-indicating blue arrowheads. d, e Rad52 foci exhibit liquid droplet behaviour $(n=6)$. White arrowheads highlight relevant behaviours. $\mathbf{f}$ Rad52 foci dynamically exchange constituents with the nucleoplasm ( $\operatorname{Rad} 52 n=30$, Tub1 $n=12$ ). Scale bars, $1 \mu \mathrm{m}$. Quantifications represent the mean \pm s.d.; ${ }^{*} P<0.0001$ in Mann-Whitney $U(\mathbf{b})$ and $\chi^{2}$ (c) tests. Source data are provided as a Source Data file.

or speculative in nature, tentatively suggest that pti-DIMs may be creating nucleoplasmic flow dynamics that drive Rad52 droplet fusion and genome stability.

Consistent with this notion, disruption of pti-DIMs upon deletion of the Tub3 $\alpha$-Tubulin isoform decreased Rad52 droplet velocities, preventing droplet clustering and hyper-inducing the DNA damage checkpoint (Fig. 4a-f; Supplementary Fig. 5a, b). Thus, pti-DIMs promote Rad52 droplet clustering and genome stability.

Rad52 droplets harbour tubulin for perinuclear DSB targeting. We then asked if the clustering of Rad52-indicated DSBs also promotes their capture by the long DIM, which then guides DSBs to the nuclear periphery. Strikingly, after rounds of Rad52 droplet fusion, when the droplet area reached the threshold size of $0.2 \mu \mathrm{m}^{2}$, these droplets internally concentrated tubulin into a focus that partially protruded from the droplets (Fig. 5a, b). Subsequently, short aster-like DIMs (aster-DIMs) emerged from the tubulin focus inside the Rad52 repair centre droplet (Fig. 5c). One aster-like DIM became dominant and transiently associated with the long DIM (Fig. 5d). The repair centre droplet then moved along the DIM to the nuclear periphery (Fig. 5e; Supplementary Movie 10), where the Rad52 focus disappeared upon repair completion $^{16}$. Thus, upon the fusion of small Rad52 droplets into a repair centre droplet, the droplet concentrates tubulin and projects aster-like microtubule filaments, which mediate repair centre capture by DIMs for perinuclear targeting and repair.

\section{Discussion}

We found that the cooperation between Rad52 liquid droplets, various nuclear filaments and possibly nucleoplasmic flow can drive the assembly and function of the DNA repair centre (Fig. 5f; Supplementary Fig. 6). However, it remains unclear as to how intranuclear microtubule filaments may generate nucleoplasmic flow. One possibility is that motor proteins slide parallel microtubule filaments relative to each other in order to achieve the observed pti-DIM extension/shortening cycles. De novo pti-DIM 


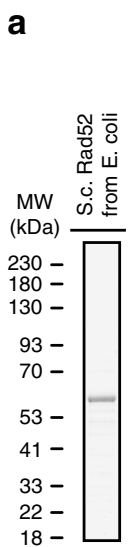

b

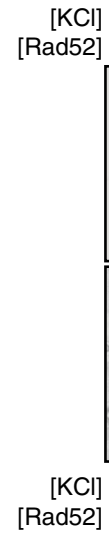

d
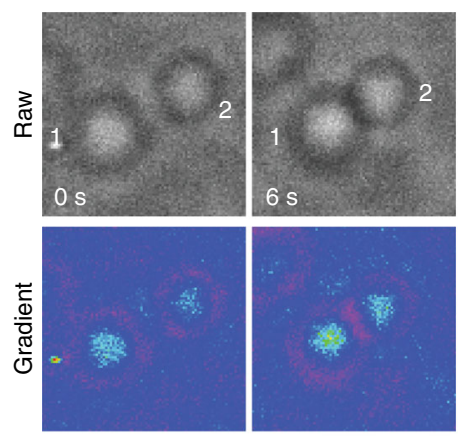

0
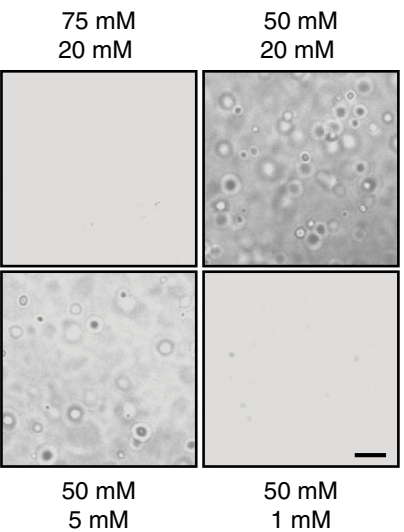

C
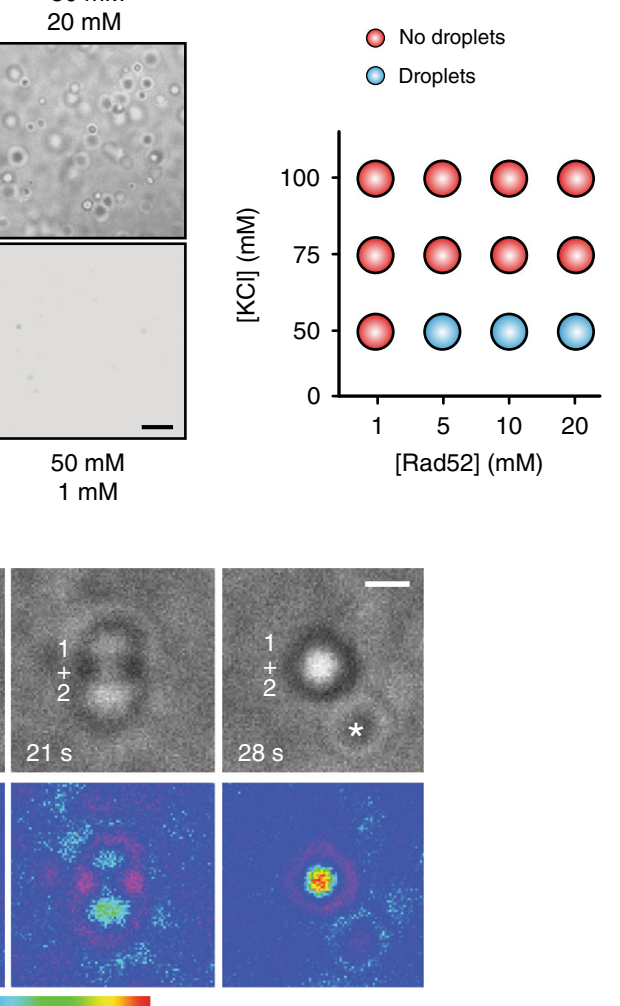

1

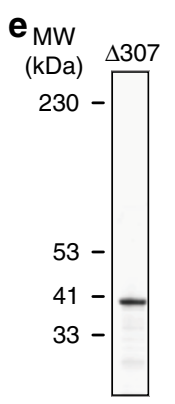

f

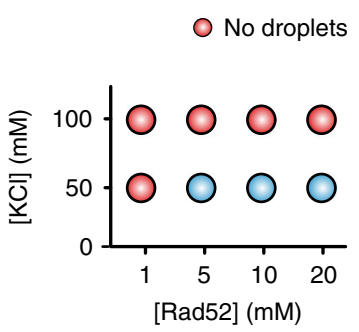

Droplets

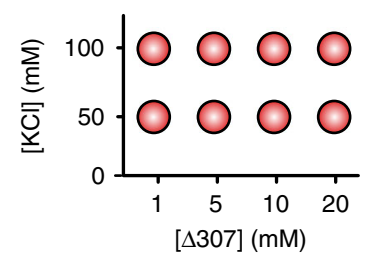

Fig. 2 Functional Rad52 forms liquid droplets in vitro. a-c Rad52 forms liquid droplets in vitro in a salt/protein concentration-dependent manner. Data represent results from experiments using three independent protein preparations. d Rad52 droplets fuse in vitro. Asterisk, separate droplet entering the imaging frame. Pseudo-colour scale, relative scale of material abundance. e, f Purified Rad52 C-terminal truncation $\Delta 307$ fails to form droplets in vitro. Scale bars, $10 \mu \mathrm{m}$ (b) and $5 \mu \mathrm{m}$ (d). Source data are provided as a Source Data file.

assembly and disassembly cycles may also contribute to the motion of the intranuclear filaments and their ability to induce a nucleoplasmic flow. Clearly, we do not observe the detachment of mobile pti-DIMs from the MTOC, arguing against a model in which pti-DIMs may be sliding through the nucleoplasm without changing in length. Future work should aim to directly explore the relative contribution of these models and the potential involvement of additional signals regulating Rad52 droplets.

Importantly, recent studies suggested that LLPS of the mammalian DNA repair protein 53BP1 can be promoted by noncoding RNA and can coordinate DNA damage responses at break sites with global p53-dependent gene activation and cell fate decisions ${ }^{27,28}$. In addition, other proteins have been observed to become liquid-like at sites of DNA damage, suggesting that coordination of the phase separation of several factors at DNA breaks may ensure global genome stability $22,24,29$. We note that another study examining irreparable DSBs did not observe a requirement for Rad52 or filaments in the clustering of such
$\mathrm{DSBs}^{30}$. Therefore, cooperation between DSB clustering and nuclear filaments in the promotion of DSB mobility likely only occurs in the context of repairable DSB systems.

Our results may also point to novel therapeutic approaches, as microtubule-dependent DSB dynamics can drive chromosome translocations and thereby alter the sensitivity of cancer cells to therapy ${ }^{7}$. Overall, we have deciphered repair centre assembly and function, expanded the repertoire of biological liquid droplets and uncovered hidden dimensions of genome stability.

\section{Methods}

Generation of yeast strains. Introduction of plasmids, gene deletions and Cterminal fluorescent tagging was done by using lithium acetate-based yeast transformation 6 . All genomic manipulations were confirmed via plating on SC drop-out medium, PCR, and/or live-cell microscopy where applicable. Yeast strains (W303 and BY4741 backgrounds) and plasmids used in this study are listed in Supplementary Table 1. Rad52-YFP and GFP-Tub1 under their endogenous promoters were expressed from a plasmid and secondary genomic locus, respectively. Forward primers used to test for deletion of KAR3 and TUB3, shown $5^{\prime}-3^{\prime}$, are 


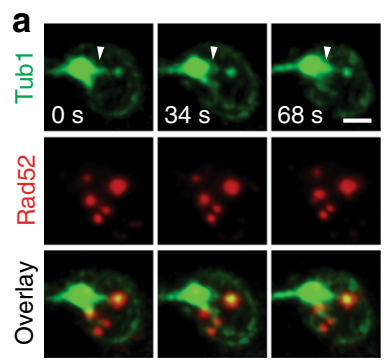

b

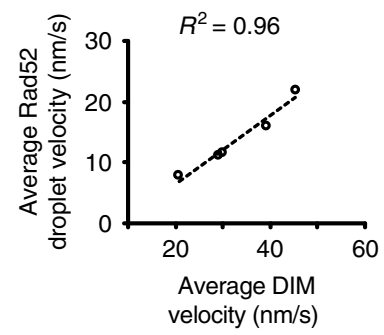

d
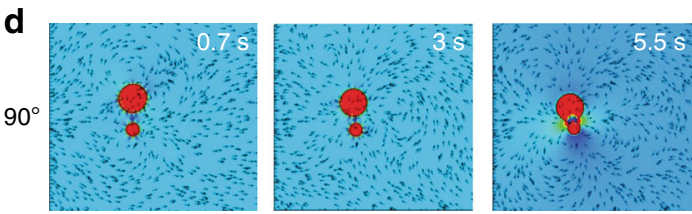

Pressure

$\begin{array}{llllll}0.005 & 0.008 & 0.011 & 0.014 & 0.017 & 0.020\end{array}$ c

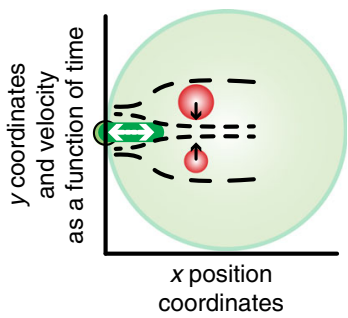

e

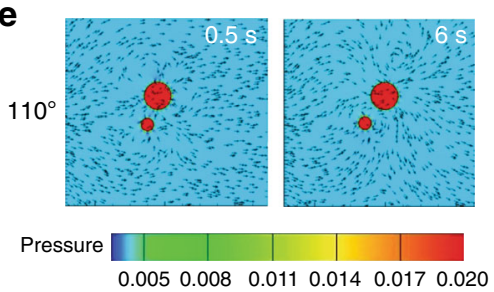

f

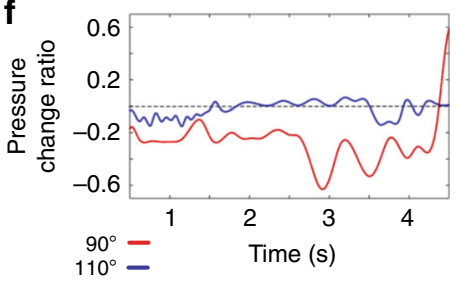

Fig. 3 Rad52 droplet fusion can be promoted by flow-generating short nuclear microtubules. $\mathbf{a}$, $\mathbf{b}$ pti-DIM extension/shortening (a, white arrowhead) correlates with Rad52 droplet velocity (a, b). c-f CFD-based modelling of pti-DIM motion shows that it can generate flow that drives Rad52 droplet fusion when pti-DIM velocity is applied at $\sim 90^{\circ}$ (d) but not at $110^{\circ}$ (e) relative to the axis connecting the centre of the droplets. Fusion is driven by an inter-droplet decrease in pressure that is shown in gradients $(\mathbf{d}, \mathbf{e})$ and quantifications $(\mathbf{f})$. Scale bar, $1 \mu \mathrm{m}$.

a

\begin{tabular}{c|c|c}
\multicolumn{3}{c}{ WT } \\
\hline Rad52 & Tub1 & Overlay \\
& 4 & \\
& & \\
\hline
\end{tabular}

b

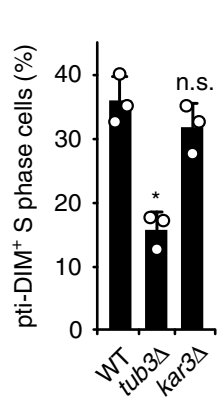

C

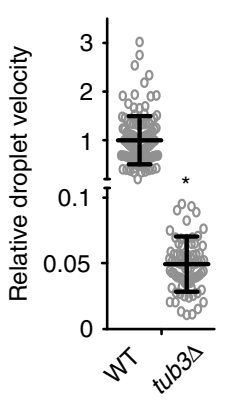

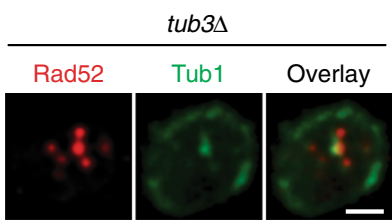

d

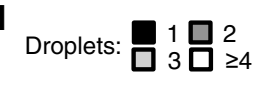

e
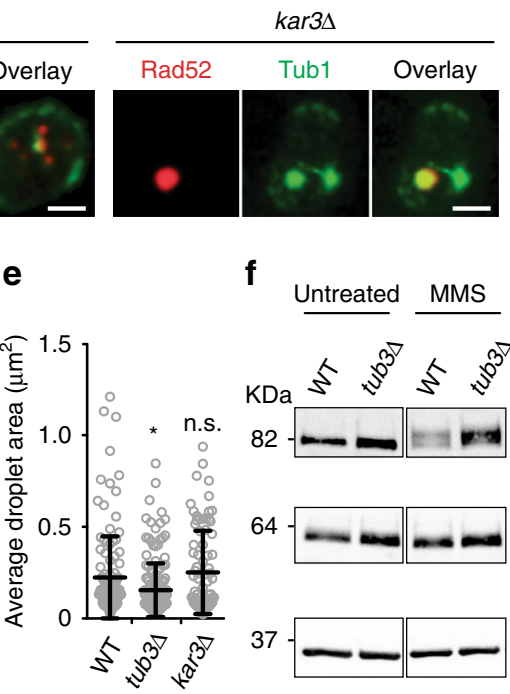

f

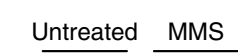

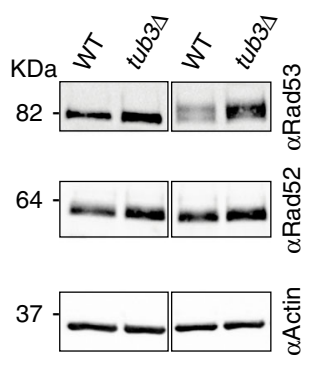

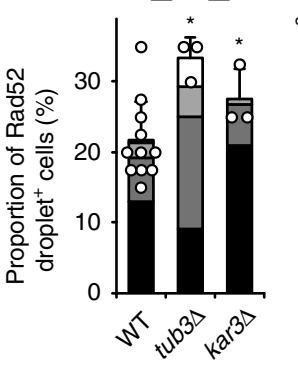

Fig. 4 pti-DIMs promote genome-stabilising clustering of Rad52 droplets. a-f Live-cell microscopy shows that the pti-DIM-compromising tub3د (a, $\mathbf{b}$ ) lowers droplet velocity (c), increases droplet numbers (d) and decreases droplet size (e), while western blotting shows Rad53 hyper-phosphorylation (f). kar3 $\Delta$ served as control. Quantifications represent the mean \pm s.d.; ${ }^{\star} P \leq 0.0004$ in $\chi^{2}(\mathbf{b}, \mathbf{d})$ and Mann-Whitney $U$ (c, e) tests. $n=3$ independent experiments, 120 cells total (b). $n=127$ (WT) and 80 droplets (tub3 $\Delta$ ) over 6 independent experiments (c). $n=11,440$ cells (WT), n=3, 120 cells each

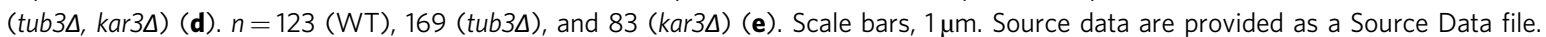

Kar3delTest (CCAATAAGAGGGTAATGGAG) and Tub3delTest (GTGCCAGA GCAGAGCATATCG). Reverse primer used to test both KAR3 and TUB3 deletion through integration of the hygromycin resistance gene is Scp164 (CTTTGTAGAA ACCATCGGCG). Forward and reverse primers used to test for C-terminal tagging of NUP49 with GFP-KanMX are NupGKanIntF (CACAGCAGATCTAGGTT CATCC) and JT57 (GACTGTCAAGGAGGGTATTC).
Microscopy. All experiments were done on logarithmic phase cells ${ }^{16}$. For drug treatment, cells were treated with $0.03 \%$ MMS or $50 \mu \mathrm{g} / \mathrm{mL}$ zeocin for $1 \mathrm{~h}$. For combined MMS and 1,6-Hexanediol treatment, cells were treated with MMS for $1 \mathrm{hr}$, pelleted and washed with $\mathrm{ddH}_{2} \mathrm{O}$, then re-suspended in SC drop-out medium with $5 \%$ 1,6-Hexanediol and digitonin $[2 \mu \mathrm{g} / \mathrm{mL}]$ for $1 \mathrm{~h}$ before imaging. Controls were treated similarly with digitonin. Small-budded S-phase cells in asynchronous 
a

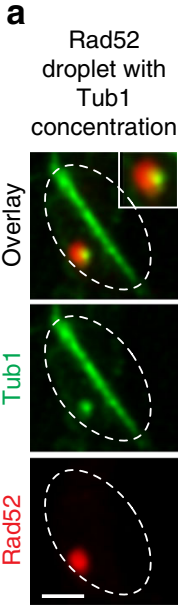

f b

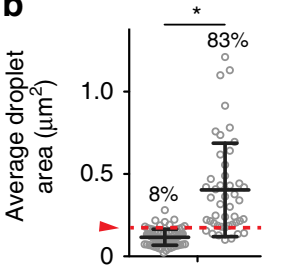

Tubulin

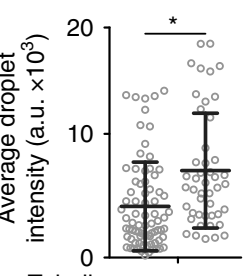

Tubulin
C Rad52
droplet with
short aster-like

MTs

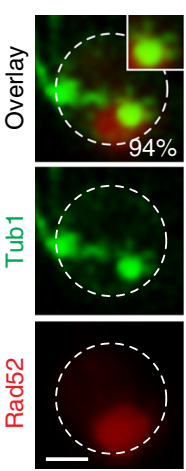

d

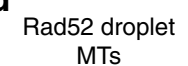
MTs connected to long MTs

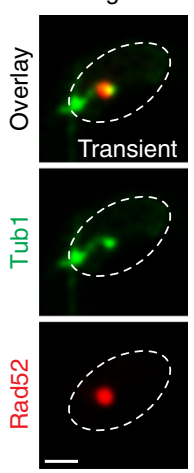

e

Long MTs capture Rad52 Droplet

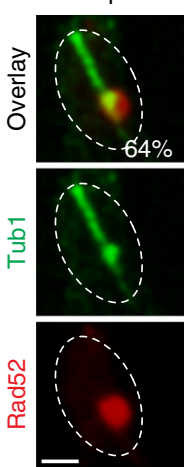

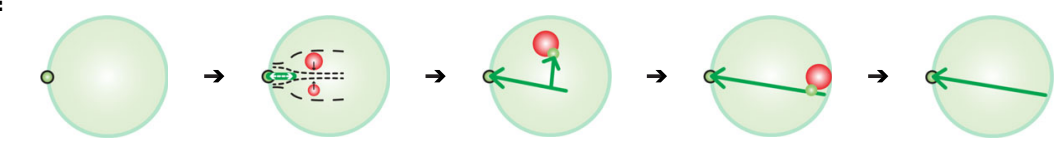

Fig. 5 Rad52 repair centre droplets concentrate tubulin and project filaments for perinuclear DSB targeting. a, b Live-cell confocal microscopy and quantification showing that Rad52 droplets exceeding $0.2 \mu \mathrm{m}^{2}$ in size (red line) have a 10-fold higher chance of concentrating tubulin ( $n=6,168$ droplets). c-e Tubulin foci inside Rad52 droplets project aster-DIMs (c) that reach the long DIM (d) before stable Rad52 droplet capture by the DIM (e). Frequency of events is shown in white. MTs microtubules. $\mathbf{f}$ Proposed model. Quantifications represent the mean \pm s.d.; ${ }^{\star} P \leq 0.0001$ in Mann-Whitney $U$ tests. $n=77$ (Tubulin-) and 46 droplets (Tubulin+) over six independent experiments (b). Scale bars, $1 \mu \mathrm{m}$. Source data are provided as a Source Data file.

cultures were subjected to live-cell confocal microscopy. Images were captured with a Leica DMI6000 SP8 LIGHTNING confocal microscope using a HC PL APO CS2 $93 \times / 1.30$ glycerol objective. Pinhole was $155.3 \mu \mathrm{m}$ and numerical aperture was 1.3 . Images were deconvolved using Leica LIGHTNING deconvolution software and processed with Leica LAS software. Cells were maintained at $30^{\circ} \mathrm{C}$ throughout imaging. GFP was excited at $458 \mathrm{~nm}$ with a laser intensity of $28 \%$ and detected with a HyD hybrid detector set to $490-523 \mathrm{~nm}$. YFP was excited at $520 \mathrm{~nm}$ with an intensity of 3\% and detected with a HyD hybrid detector set to 525-661 nm. For observation of liquid droplet behaviours, $\mathrm{z}$-stack time-lapses were taken for 5-10 min. For Fig. 1d, $n=6$ independent experiments. Each one of these six replicates consisted of the analysis of 30 cells that together provide $\geq 168$ droplets. Cells were counted once. Maximum intensity projections are shown but all findings were confirmed on a single-plane. The time-lapse imaging was used to detect droplet fusion (droplets hit each other, elongate, then relax into spherical shape), bumping (droplets hit each other but bounce off), dripping (droplets approach each other and establish an elongated connection but then separate) and wetting (droplets conform to the shape of neighbouring surfaces). Particle velocities were measured using Imaris image analysis software.

FLIP. Yeast strain KMY3426 was treated with $0.03 \%$ MMS and subjected to live-cell confocal microscopy as described above. Images were acquired with a Leica SP8 LIGHTNING confocal microscope using an excitation wavelength of $488 \mathrm{~nm}$, laser power of $0.2 \%$ and an HC PL APO CS2 $\times 63 / 1.40$ oil objective. GFP and YFP signals were detected and separated using HyD hybrid detectors set to 493-511 nm and $541-638 \mathrm{~nm}$ respectively. Pinhole was $95.5 \mu \mathrm{m}$ and numerical aperture was 1.4. FLIP setup and subsequent analysis was done using Leica LAS software. For FLIP, $\mathrm{S}$ phase cells with Rad52-YFP foci were imaged 25 times before bleaching. A nuclear point outside the focus was bleached at $15 \%$ laser power for $25 \mathrm{~ms}$ and the cell was subsequently imaged. This bleaching/imaging process was repeated 25 times. The intensity of the focus was monitored throughout and normalised to the peak focus intensity.

Protein isolation and immunoblotting. Briefly, $2.0 \times 10^{7}$ cells were pelleted and protein was isolated and immunoblotting was performed ${ }^{6}$. Rad53 and Rad52 were detected using anti-Rad53 (Abcam-ab104232) and anti-Rad52 (gift from B. Pfander) antibodies, both diluted 1/10,000. Actin was detected using anti-Actin antibody (ThermoFisher-MA1-744) diluted 1/5000. Chemiluminescence was captured using autoradiography and VersaDoc imager to ensure ideal exposure in linear range.

Expression and purification of Rad52, $\Delta \mathbf{3 0 7}$, and RPA. Rad52 harbouring a Cterminal hexahistidine tag was expressed from plasmid pET11d, a kind gift from Dr. Lumir Krejci ${ }^{23}$. The $\Delta 307$ mutant was cloned from this template using PCR and standard methods and verified by sequencing. Rad52-His6 was expressed in $E$. coli BL21 Star pRARE. Luria broth (LB) cultures supplemented with $100 \mu \mathrm{g} / \mathrm{mL}$ ampicillin and $25 \mu \mathrm{g} / \mathrm{mL}$ chloramphenicol were inoculated with a single cuolony from a freshly transformed plate, grown overnight at $37^{\circ} \mathrm{C}$, and diluted 100 -fold into fresh LB containing $100 \mu \mathrm{g} / \mathrm{mL}$ ampicillin, $25 \mu \mathrm{g} / \mathrm{mL}$ chloramphenicol and incubated at $37^{\circ} \mathrm{C}$ until OD600 0.6. Cultures were subsequently incubated at $16^{\circ} \mathrm{C}$ to an $\mathrm{OD}_{600} \sim 0.8-1$. Expression of Rad52-His6 was induced by the addition of $0.1 \mathrm{mM}$ isopropyl-D-thiogalactopyranoside and cultures were incubated at $16^{\circ} \mathrm{C}$ for 21-24 h. Purification of Rad52-His6 was performed ${ }^{23}$. The purified protein was dialysed against storage buffer ( $50 \mathrm{mM}$ Tris- $\mathrm{Cl} \mathrm{pH} \mathrm{7.5,100} \mathrm{mM} \mathrm{KCl,} 10 \%$ glycerol, $1 \mathrm{mM}$ EDTA). As an alternative strategy, gel filtration through a Superdex 200 Increase 10/300 GL (GE Healthcare) column was used instead of hydroxyapatite chromatography ${ }^{23}$. The $\Delta 307$-His6 protein ${ }^{25}$ was produced similarly, with the following exceptions: (i) cultures were grown in Terrific broth (TB) and (ii) the final step of the purification involved gel filtration through a Superdex 200 Increase 10/300 GL (GE Healthcare) column in storage buffer $(50 \mathrm{mM}$ Tris-Cl pH 7.5, $100 \mathrm{mM} \mathrm{KCl}, 10 \%$ glycerol, $1 \mathrm{mM} \mathrm{EDTA}$ ), instead of hydroxyapatite purification. Aliquots of all proteins were snap-frozen in liquid $\mathrm{N}_{2}$ and stored at $-80^{\circ} \mathrm{C}$. The Rpa heterotrimer was expressed from the pET11d plasmid ${ }^{26}$. Rpa was expressed and purified from E. coli BL21 Star pRARE cultures grown in $\mathrm{TB}^{31}$.

In vitro droplet assembly. Purified Rad52 protein was concentrated using an Amicon Ultra- $0.5 \mathrm{~mL}$ centrifugal filter and suspended in glycerol-free storage buffer (50 mM Tris-Cl pH 7.5, $100 \mathrm{mM} \mathrm{KCl,} 1 \mathrm{mM}$ EDTA). RPA and ssDNA complexes were generated in vitro by incubating $10 \mu \mathrm{M}$ of purified RPA and $100 \mu \mathrm{M}$ of PAGE-purified Oligo $\mathrm{dT}_{30}$ (IDT) in RPA storage buffer (60 mM HEPES $\mathrm{pH}$ 7.5, $0.5 \%$ Hyoinositol, $0.02 \%$ Tween $20,0.5 \mathrm{mM}$ EDTA) for $30 \mathrm{~min}$ at room temperature ${ }^{32}$. In total, $20 \mu \mathrm{L}$ reactions were performed in an uncoated 384-well coverslip plate (MatTek). Protein and glycerol-free dialysis buffer $(50 \mathrm{mM}$ Tris-Cl $\mathrm{pH} 7.5,100 \mathrm{mM} \mathrm{KCl}, 1 \mathrm{mM}$ EDTA) and/or salt-free glycerol-free dialysis buffer (50 mM Tris-Cl pH 7.5, $1 \mathrm{mM}$ EDTA) were added to indicated concentrations. Reactions were imaged immediately following assembly. Images were captured with a Nikon C2+Confocal Microscope using a Plan-Apochromat TIRF 60x oil objective, numerical aperture of 1.4 and pinhole of $30.0 \mu \mathrm{m}$. Phase separation in vitro was confirmed under indicated conditions using three independent Rad52 protein preparations.

CFD: In order to simulate the dynamics of liquid droplets, the following governing equations of mass and momentum are solved using ANSYS-Fluent (version 16.2):

$$
\begin{gathered}
\frac{1}{\rho_{q}}\left[\frac{\partial}{\partial t}\left(a_{q} \rho_{q}\right)+\nabla \cdot\left(a_{q} \rho_{q} \vec{u}_{q}\right)=0\right] \\
\frac{\partial}{\partial t}(\rho \vec{u})+\nabla \cdot(\rho \vec{u} \vec{u})=-\nabla p+\nabla \cdot\left[\mu\left(\nabla \vec{u}+\nabla \vec{u}^{\mathrm{T}}\right)\right]+F
\end{gathered}
$$

where, $a$ represents the volume fraction of a phase, with $\sum_{q=1}^{n} a_{q}=1$, where $q$ is a phase indicator ( 1 for the continuous phase, and 2 for the droplet), $\rho$ is the 
volume average density and it is calculated by $\rho=a_{1} \rho_{1}+\left(1-a_{1}\right) \rho_{2}, u$ is velocity, $t$ is time, $p$ is pressure, $\mu$ is viscosity and it is calculated by $\mu=a_{1} \mu_{1}+\left(1-a_{1}\right) \mu_{2}$ and $F$ is an external force. Continuum surface tension force (CSF) model is applied to model the surface tension force on the droplet interface. In this model, the pressure at the interphase is defined by $P_{2}-P_{1}=2 \sigma / R$, where $R$ is the radius of the curvature $(\kappa)$. The curvature is defined by the unit surface normal vector, where the normal vector is defined as the gradient of the volume fraction of the secondary phase, $n=\nabla a_{q}: \kappa=\nabla$. , where $\hat{n}=n /|n|$. The surface tension force is $F=2 \sigma \rho \kappa \nabla a /\left(\rho_{1}+\rho_{2}\right)$. This force is then inserted in equations above, which are solved numerically. Pressure and velocity are coupled with SIMPLE method. Pressure and momentum discretization methods are PRESTO and Second Order Upwind, respectively. Time is discretized by Second Order Implicit method. GeoReconstruction method is applied for the volume fraction discretization. Inlet velocity is calculated as $U=A \cos \omega \mathrm{t}$, where $\omega=2 \pi \mathrm{f}, A$ is average velocity, $\omega$ is angular frequency and $\mathrm{f}$ is frequency. The computational domain used consisted of 398,866 elements and the time interval was initially set to $10^{-8}$ s and was later adjusted by local courant number.

Statistical analysis. Mann-Whitney $U$ and $\chi^{2}$ square tests were used to compare non-normally distributed and categorical datasets respectively using GraphPad Prism 7.

Reporting summary. Further information on research design is available in the Nature Research Reporting Summary linked to this article.

\section{Data availability}

All data are in the paper and supplementary information. The source data underlying Figs. 1a-f, 2a, e, 4b-f, 5b and Supplementary Figs. 1a, b, e, 2c, 3a, b and 5a, b are provided as a Source Data file. All data is available from the authors upon reasonable requests.

Received: 12 September 2019; Accepted: 17 January 2020;

Published online: 04 February 2020

\section{References}

1. Mekhail, K. \& Moazed, D. The nuclear envelope in genome organization, expression and stability. Nat. Rev. Mol. Cell Biol. 11, 317-328 (2010).

2. Sanchez, Y. et al. Regulation of RAD53 by the ATM-like kinases MEC1 and TEL1 in yeast cell cycle checkpoint pathways. Science 271, 357-360 (1996).

3. Aymard, F. et al. Genome-wide mapping of long-range contacts unveils clustering of DNA double-strand breaks at damaged active genes. Nat. Struct. Mol. Biol. 24, 353-361 (2017).

4. Lawrimore, J. et al. Microtubule dynamics drive enhanced chromatin motion and mobilize telomeres in response to DNA damage. Mol. Biol. Cell 28, 1701-1711 (2017).

5. Tsouroula, K. et al. Temporal and spatial uncoupling of DNA double strand break repair pathways within mammalian heterochromatin. Mol. Cell 63 , 293-305 (2016).

6. Chung, D. K. et al. Perinuclear tethers license telomeric DSBs for a broad kinesin- and NPC-dependent DNA repair process. Nat. Commun. 6, 1-13 (2015).

7. Lottersberger, F., Karssemeijer, R. A., Dimitrova, N. \& De Lange, T. 53BP1 and the LINC complex promote microtubule-dependent DSB mobility and DNA repair. Cell 163, 880-893 (2015).

8. Ryu, T. et al. Heterochromatic breaks move to the nuclear periphery to continue recombinational repair. Nat. Cell Biol. 17, 1401-1411 (2015).

9. Dion, V., Kalck, V., Horigome, C., Towbin, B. D. \& Gasser, S. M. Increased mobility of double-strand breaks requires $\mathrm{Mec} 1, \operatorname{Rad} 9$ and the homologous recombination machinery. Nat. Cell Biol. 14, 502-509 (2012).

10. Miné-Hattab, J. \& Rothstein, R. Increased chromosome mobility facilitates homology search during recombination. Nat. Cell Biol. 14, 510-517 (2012)

11. Chiolo, I. et al. Double-strand breaks in heterochromatin move outside of a dynamic HP1a domain to complete recombinational repair. Cell 144, 732-744 (2011).

12. Khadaroo, B. et al. The DNA damage response at eroded telomeres and tethering to the nuclear pore complex. Nat. Cell Biol. 11, 980-987 (2009).

13. Oza, P., Jaspersen, S. L., Miele, A., Dekker, J. \& Peterson, C. L. Mechanisms that regulate localization of a DNA double-strand break to the nuclear periphery. Genes Dev. 23, 912-927 (2009).

14. Dimitrova, N., Chen, Y.-C. M. C., Spector, D. L. \& De Lange, T. 53BP1 promotes non-homologous end joining of telomeres by increasing chromatin mobility. Nature 456, 524-528 (2008).

15. Torres-Rosell, J. et al. The Smc5-Smc6 complex and SUMO modification of Rad52 regulates recombinational repair at the ribosomal gene locus. Nat. Cell Biol. 9, 923-931 (2007).
16. Oshidari, R. et al. Nuclear microtubule filaments mediate non-linear directional motion of chromatin and promote DNA repair. Nat. Commun. 9, 2567 (2018)

17. Schrank, B. R. et al. Nuclear ARP2/3 drives DNA break clustering for homology-directed repair. Nature 559, 61-66 (2018).

18. Caridi, C. P. et al. Nuclear F-actin and myosins drive relocalization of heterochromatic breaks. Nature 559, 54-60 (2018).

19. Lisby, M., Mortensen, U. H. \& Rothstein, R. Colocalization of multiple DNA double-strand breaks at a single Rad52 repair centre. Nat. Cell Biol. 5, 527-577 (2003).

20. Ashgriz, N. \& Poo, J. Y. Coalescence and separation in binary collisions of liquid-drops. J. Fluid Mech. 221, 183-204 (1990).

21. Orme, M. Experiments on droplet collisions, bounce, coalescence and disruption. Prog. Energy Combust. Sci. 23, 65-79 (1997).

22. Banani, S. F., Lee, H. O., Hyman, A. A. \& Rosen, M. K. Biomolecular condensates: organizers of cellular biochemistry. Nat. Rev. Mol. Cell Biol. 18, 285-298 (2017).

23. Seong, C. et al. Molecular anatomy of the recombination mediator function of Saccharomyces cerevisiae Rad52. J. Biol. Chem. 283, 12166-12174 (2008).

24. Patel, A. et al. A liquid-to-solid phase transition of the ALS protein FUS accelerated by disease mutation. Cell 162, 1066-1077 (2015).

25. Plate, I. et al. Interaction with RPA is necessary for Rad52 repair center formation and for its mediator activity. J. Biol. Chem. 283, 29077-29085 (2008).

26. Sibenaller, Z. A., Sorensen, B. R. \& Wold, M. S. The 32- and 14-kilodalton subunits of replication protein $A$ are responsible for species-specific interactions with single-stranded DNA. Biochemistry 37, 12496-12506 (1998).

27. Kilic, S., et al. Phase separation of 53BP1 determines liquid-like behavior of DNA repair compartments. EMBO J. 38, e101379 (2019).

28. Pessina, F. et al. Functional transcription promoters at DNA double-strand breaks mediate RNA-driven phase separation of damage-response factors. Nat. Cell Biol. 21, 1286-1299 (2019).

29. Altmeyer, M. et al. Liquid demixing of intrinsically disordered proteins is seeded by poly(ADP-ribose). Nat. Commun. 6, 8088 (2015)

30. Waterman, D. P. et al. Live cell monitoring of double strand breaks in S. cerevisiae. PLoS Genet. 15, el008001 (2019).

31. Binz, S. K., Dickson, A. M., Haring, S. J. \& Wold, M. S. Functional assays for replication protein A (RPA). Methods Enzymol. 409, 11-38 (2006)

32. Kumaran, S., Kozlov, A. G. \& Lohman, T. M. Saccharomyces cerevisiae replication protein A binds to single-stranded DNA in multiple saltdependent modes. Biochemistry 45, 11958-11973 (2006).

\section{Acknowledgements}

We thank the D. Durocher, G. Brown and R. Hakem labs for reagents and discussions We thank B. Pfander and U. Mortensen for reagents and strains. R.O. is supported by a Doctoral Scholarship from the Natural Sciences and Engineering Research Council of Canada (NSERC). The work was supported by a Canadian Institutes of Health Research (CIHR) grant (156297) to H.W., who holds the Canada Research Chair in Mechanisms of Genome Instability (950-231487). The work was mainly supported by funding from the CIHR $(388041,399687)$ and the Ontario Ministry of Research and Innovation (MRIERA; ER13-09-111) to K.M., who holds the Canada Research Chair in Spatial Genome Organization (CRC; 950-230661)

\section{Author contributions}

R.O. and K.M. conceived the study and wrote the paper. Text editing by all co-authors. R O. was the lead on all experiments except for the bacterial expression system (H.W. with E.Y.W.T) and in vitro droplet assays (R.O. and H.O.L.). M.M. executed CFD analyses under the guidance of N.A. and K.M.; R.H. contributed to immunoblotting, strain making and microscopy.

\section{Competing interests}

The authors declare no competing interests.

\section{Additional information}

Supplementary information is available for this paper at https://doi.org/10.1038/s41467 020-14546-z.

Correspondence and requests for materials should be addressed to K.M.

Peer review information Nature Communications thanks the anonymous reviewer(s) for their contribution to the peer review of this work.

Reprints and permission information is available at http://www.nature.com/reprints

Publisher's note Springer Nature remains neutral with regard to jurisdictional claims in published maps and institutional affiliations. 
(c) (i) Open Access This article is licensed under a Creative Commons Attribution 4.0 International License, which permits use, sharing, adaptation, distribution and reproduction in any medium or format, as long as you give appropriate credit to the original author(s) and the source, provide a link to the Creative Commons license, and indicate if changes were made. The images or other third party material in this article are included in the article's Creative Commons license, unless indicated otherwise in a credit line to the material. If material is not included in the article's Creative Commons license and your intended use is not permitted by statutory regulation or exceeds the permitted use, you will need to obtain permission directly from the copyright holder. To view a copy of this license, visit http://creativecommons.org/ licenses/by/4.0/.

(C) The Author(s) 2020 